\title{
Sine-Gordon on a wormhole
}

\author{
Piotr Bizoń ${ }^{1, *}$, Maciej Dunajski ${ }^{2}$, Michał Kahl ${ }^{1}$ and \\ Michał Kowalczyk ${ }^{3}$
}

${ }^{1}$ Institute of Physics, Jagiellonian University, Kraków, Poland

2 DAMTP, University of Cambridge, Cambridge, United Kingdom

3 Departamento de Ingeniería Matemática and Centro de Modelamiento Matemático, Universidad de Chile, Santiago, Chile

E-mail: bizon@th.if.uj.edu.pl,m.dunajski@damtp.cam.ac.uk, michal.kahl@alumni.uj.edu.pl and kowalczy@dim.uchile.cl

Received 23 December 2020, revised 25 May 2021

Accepted for publication 7 June 2021

Published 2 July 2021

\section{Abstract}

In an attempt to understand the soliton resolution conjecture, we consider the sine-Gordon equation on a spherically symmetric wormhole spacetime. We show that within each topological sector (indexed by a positive integer degree $n$ ) there exists a unique linearly stable soliton, which we call the $n$-kink. We give numerical evidence that the $n$-kink is a global attractor in the evolution of any smooth, finite energy solutions of degree $n$. When the radius of the wormhole throat $a$ is large enough, the convergence to the $n$-kink is shown to be governed by internal modes that slowly decay due to the resonant transfer of energy to radiation. We compute the exact asymptotics of this relaxation process for the one-kink using the Soffer-Weinstein weakly nonlinear perturbation theory.

Keywords: soliton resolution conjecture, asymptotic stability of solitons, nonlinear dispersive equations

Mathematics Subject Classification numbers: 35C08.

(Some figures may appear in colour only in the online journal)

\section{Introduction}

If a solution of an evolution equation exists for all times $t$, then it is natural to ask how it behaves as $t \rightarrow \infty$. This question is particularly interesting if the equation admits solitons (spatially localised, finite energy solutions) because they may appear as late-time attractors. For

*Author to whom any correspondence should be addressed.

Recommended by Dr Christian Klein.

Original content from this work may be used under the terms of the Creative Commons Attribution 3.0 licence. Any further distribution of this work must maintain attribution to the author(s) and the title of the work, journal citation and DOI. 

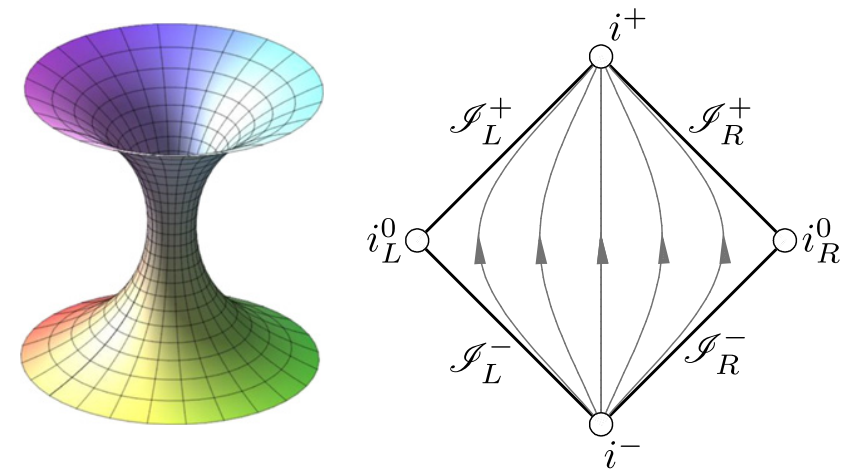

Figure 1. (Left panel) The isometric embedding of the constant time equatorial crosssection of the wormhole in $\mathbb{R}^{3}$. (Right panel) The conformal diagram of the wormhole spacetime. The boundaries of each side of the diamond, denoted by $\mathcal{J}_{\mathrm{L}, \mathrm{R}}^{ \pm}$, represent future/past $(t \rightarrow \pm \infty)$ and left/right $(r \rightarrow \mp \infty)$ null infinities.

nonlinear dispersive wave equations it is believed that for any reasonable (e.g. smooth and finite energy) generic initial data, the solution eventually resolves into a superposition of a radiative component plus a finite number of solitons. This belief, known as the soliton resolution conjecture [1], is fairly well understood for small perturbations of solitons [2, 3], however little is known in the non-perturbative regime (but the one-dimensional completely integrable equations where solutions can be computed explicitly via inverse scattering methods $[4,5]$ and few results in higher dimensions, e.g. [6, 7]).

In an attempt to understand the soliton resolution conjecture in a simple setting, two of us proposed in [8] to study nonlinear waves propagating on a spherically symmetric curved spacetime with the metric

$$
\mathrm{d} s^{2}=-\mathrm{d} t^{2}+\mathrm{d} r^{2}+\left(r^{2}+a^{2}\right) \mathrm{d} \omega^{2}
$$

where $(t, r) \in \mathbb{R}^{2}, \mathrm{~d} \omega^{2}$ is the round metric on the unit two-sphere, and $a$ is a positive constant. This spacetime, introduced by Ellis [9] and Bronnikov [10], is the simplest example of a wormhole geometry that has two asymptotically flat ends at $r \rightarrow \pm \infty$ connected by a spherical throat (minimal surface) of area $4 \pi a^{2}$ at $r=0$ (figure 1).

Although the wormhole belongs more to science-fiction than physics [11], it has a number of features that makes it an attractive testing ground for the soliton resolution conjecture. First, there is no singularity at $r=0$ which basically ensures global well-posedness for dispersive equations with coercive nonlinearities. Second, due to the presence of the length scale $a$, Derrick's non-existence scaling arguments are evaded and solitons do exist (often in abundance) for some nonlinearities. Third, if a soliton exists, it is completely rigid so no modulation analysis is needed. Finally, the equations posed on the wormhole combine the simplicity of one-dimensional equations on the whole real line with the three-dimensional dispersive properties.

In [8] the soliton resolution conjecture was formulated and verified numerically for equivariant wave maps from the wormhole (1) into the three-sphere. In addition, the rate of convergence to the soliton (which in this case is a harmonic map from a $t=$ const. hypersurface of the wormhole into the three-sphere) was computed by perturbation methods. Subsequently, the conjecture made in [8] was proved (without a decay rate, though) by Rodriguez $[12,13]$ via the concentration-compactness method as in [7]. The key ingredient in getting these results was 
the fact that the linearised perturbations around the solitons decay in time. In this paper, we consider a different nonlinearity for which the latter property does not hold and the asymptotic stability of solitons is an inherently nonlinear phenomenon.

On the wormhole spacetime we consider a real scalar field $\phi$ obeying the semilinear wave equation $^{4}$

$$
\square_{g} \phi+\sin (2 \phi)=0,
$$

where $\square_{g}$ is the wave operator associated with the metric (1). Assuming that $\phi=\phi(t, r)$, we get

$$
\ddot{\phi}=\phi^{\prime \prime}+\frac{2 r}{r^{2}+a^{2}} \phi^{\prime}-\sin (2 \phi),
$$

where an overdot and prime denote derivatives with respect to $t$ and $r$. For $a=\infty$ this equation reduces to the one-dimensional sine-Gordon equation

$$
\ddot{\phi}=\phi^{\prime \prime}-\sin (2 \phi)
$$

which is completely integrable, hence for large values of $a$ equation (3) can be viewed as a non-integrable perturbation of (4). ${ }^{5}$

The conserved energy associated with equation (3) reads

$$
E=\int_{-\infty}^{\infty}\left(\frac{1}{2} \dot{\phi}^{2}+\frac{1}{2} \phi^{\prime 2}+\sin ^{2} \phi\right)\left(r^{2}+a^{2}\right) \mathrm{d} r .
$$

Finiteness of energy requires that $\phi(t,-\infty)=n_{-} \pi, \phi(t, \infty)=n_{+} \pi$, where $n_{-}$and $n_{+}$are integers. Without loss of generality we choose $n_{-}=0$; then $n=n_{+}$determines the topological degree of the solution (which is preserved in the evolution).

The goal of this paper is to describe the asymptotic behaviour of solutions of equation (3) for $t \rightarrow \infty$. Due to the dissipation of energy by dispersion, solutions are expected to settle down to stationary states, in accord with the soliton resolution conjecture. In section 2 we prove that for each degree $n$ there exists a unique smooth, finite-energy stationary solution, which we call the $n$-kink. The linear stability of the $n$-kinks is analysed in section 3 . We show that the spectrum of the linearised operator around the $n$-kink has no negative or zero eigenvalues, hence the $n$-kink is linearly stable. However, for sufficiently large $a$ there are $n$ positive eigenvalues in the mass gap between zero and the bottom of the continuous spectrum. These positive eigenvalues give rise to internal modes that oscillate harmonically and therefore prevent asymptotic stability of kinks at the linear level (if $a$ is large enough). Nonetheless, it is expected that the $n$-kink is asymptotically stable thanks to the nonlinear resonant damping of internal modes, as described by Soffer and Weinstein in [15]. In section 4 we use their weakly nonlinear perturbation method to derive the decay rate of the internal mode for the one-kink. Finally, in section 5 we give numerical evidence for the soliton resolution conjecture and verify the predictions of perturbative computations. As in [8], we solve equation (3) numerically using the hyperboloidal formulation of the initial value problem. This approach allows us to reach very long

\footnotetext{
${ }^{4}$ For dimensional reasons the nonlinear term must have the form $\ell^{-2} \sin (2 \phi)$, where $\ell$ is a fixed scale of length. Hereafter, we set $\ell=1$ by the choice of the unit of length.

${ }^{5}$ The $\phi^{4}$ model on the wormhole was considered in [14] following an earlier unpublished version of our paper. The key difference which makes the $\phi^{4}$ model less interesting than sine-Gordon is that the former is non-integrable already in flat space. Equation (3) is indeed not integrable for any finite $a$ as it does not possess the Painlevé property.
} 
times of evolution in a reasonable computational time. In the appendix we give some details of the computation of parameters of kinks.

\section{Kinks}

Time-independent solutions $\phi=\phi(r)$ of equation (3) satisfy the ordinary differential equation

$$
\phi^{\prime \prime}+\frac{2 r}{r^{2}+a^{2}} \phi^{\prime}-\sin (2 \phi)=0
$$

This equation can be viewed as the equation of motion, with 'time' $r$, for the unit mass particle moving in the potential $-\sin ^{2} \phi$ and subject to friction with the time-dependent friction coefficient $\frac{2 r}{r^{2}+a^{2}}$. The solution of degree $n$ corresponds to the trajectory whose projection on the phase plane $\left(\phi, \phi^{\prime}\right)$ starts from the saddle point $(0,0)$ at $r=-\infty$ and goes to the saddle point $(n \pi, 0)$ for $r=+\infty$. The existence and uniqueness of such a connecting trajectory for each $n$ follows from an elementary shooting argument. For example, let the particle be located at $\phi=\pi / 2$ for $r=0$. If the velocity $b=\phi^{\prime}(0)$ is too small, then the particle will never reach the hilltop at $\phi=\pi$, while if $b$ is sufficiently large it will roll over the hilltop. By continuity, there must be a critical velocity $b_{1}$ for which the particle reaches the hilltop in infinite time (obviously, by the uniqueness of trajectories, the particle cannot stop at the hilltop in finite time). Due to reflection symmetry $r \rightarrow-r$, the particle sent backwards in time reaches $\phi=0$ for $r \rightarrow-\infty$, giving the desired connecting trajectory with $n=1 .{ }^{6}$ Repeating this argument for higher $n$ we get a countable family of unique connecting trajectories $\phi_{n}(r)$ which are symmetric with respect to the midpoint $\phi(0)=n \pi / 2$, that is $\phi_{n}(r)+\phi_{n}(-r)=n \pi$. Near $r=0$

$$
\phi_{n}(r)=\frac{n \pi}{2}+b_{n} r+\mathcal{O}\left(r^{3}\right)
$$

where the parameter $b_{n}$ uniquely determines the trajectory. For $r \rightarrow \mp \infty$ the leading asymptotics are, respectively

$$
\phi_{n}(r) \sim-\frac{c_{n}}{r} \mathrm{e}^{\sqrt{2} r} \quad \text { and } \quad \phi_{n}(r) \sim n \pi-\frac{c_{n}}{r} \mathrm{e}^{-\sqrt{2} r}
$$

where the parameter $c_{n}$ is determined by $b_{n}$. In the following, we shall refer to the stationary solution $\phi_{n}$ as the $n$-kink. Figure 2 depicts sample profiles of $n$-kinks for $n=1,2$. A few values of the parameters $b_{n}$ and $c_{n}$ are listed in table 1 for different values of $a$ and $n$. While the numerical computation of the parameters $b_{n}$ is straightforward by means of the shooting method, the computation of the parameters $c_{n}$ is more difficult because the leading asymptotic behaviour (8) is only the first term of the asymptotic series which has to be summed to give an accurate approximation of the solution. The details of this computation are given in the appendix.

Next, we shall derive analytic approximations of the kink solutions for large and small values of $a$. These approximations will be used below in the stability analysis of the kink.

Large $a$ approximation: for $a=\infty$, equation (6) reduces to the static sine-Gordon equation

$$
\phi^{\prime \prime}-\sin (2 \phi)=0
$$

\footnotetext{
${ }^{6}$ If $0<\phi(0)<\pi / 2$, then by the same shooting argument there exists a velocity $\phi^{\prime}(0)$ such that the particle tends to $\pi$ as $r \rightarrow \infty$, however going backwards this particle will overshoot 0 and end up at $-\pi / 2$ for $r \rightarrow-\infty$. Thus, there are no asymmetric kinks.
} 

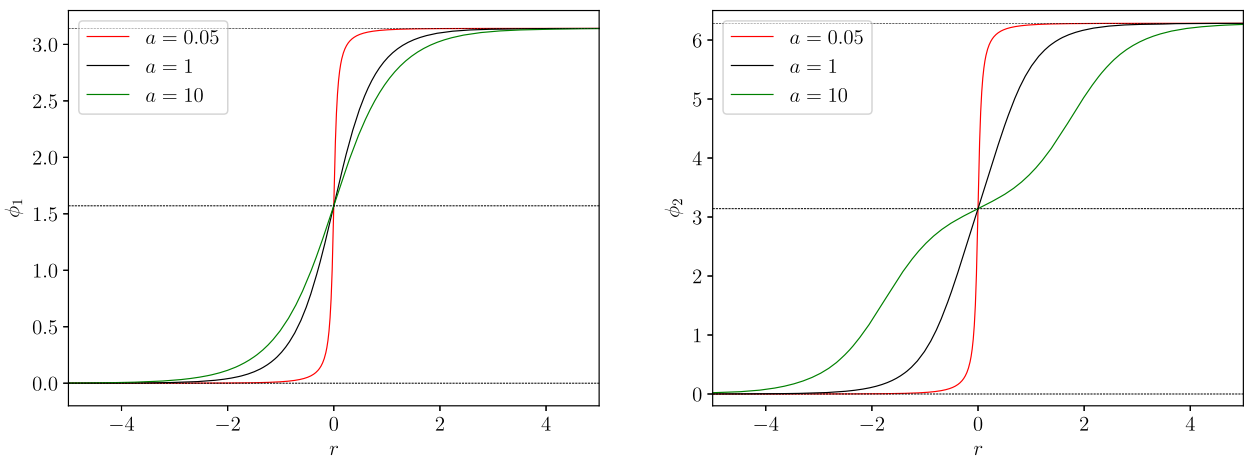

Figure 2. Profiles of the $n$-kinks for $n=1,2$.

Table 1. Parameters $b_{n}$ and $c_{n}$ for three values of $a$.

\begin{tabular}{cccc}
\hline$a$ & $\left(b_{1}, c_{1}\right)$ & $\left(b_{2}, c_{2}\right)$ & $\left(b_{3}, c_{3}\right)$ \\
\hline 1 & $(2.0163,1.5054)$ & $(2.8709,4.2523)$ & $(4.3285,8.5162)$ \\
2 & $(1.6152,3.4063)$ & $(1.6531,13.109)$ & $(2.7121,33.218)$ \\
3 & $(1.5123,5.3885)$ & $(1.1993,26.592)$ & $(2.1862,82.056)$ \\
\hline
\end{tabular}

whose unique (modulo translation) soliton solution is the sine-Gordon kink

$$
H(r)=2 \arctan \mathrm{e}^{\sqrt{2} r} .
$$

Let $\varepsilon=1 / a \ll 1$ and write

$$
\phi(r)=H(r)+\varepsilon^{2} \psi(r)+\mathcal{O}\left(\varepsilon^{4}\right) .
$$

Substituting this expansion into equation (6) and collecting terms of order $\varepsilon^{2}$, we obtain

$$
\psi^{\prime \prime}-2 \cos (2 H) \psi=-2 r H^{\prime} .
$$

The solution $H^{\prime}$ of the homogeneous equation is even, while the right-hand side is odd, hence the Fredholm solvability condition is satisfied and consequently there is a unique solution that is odd and decays at $\pm \infty$. This solution can be written in closed form (using a polylogarithmic function) but we refrain from displaying it here because it will not be used below.

We confirmed numerically that the approximation $\phi_{1}(r) \approx H(r)+\psi(r) / a^{2}$ is very accurate for sufficiently large $a^{2}$ (and $r^{2}<a^{2}$ ).

The $n$-kink for large $a$ can be approximated by a superposition of $n$ well-separated sineGordon kinks. For example, for $n=2$ we have

$$
\phi_{2}(r) \approx H(r-R)+H(r+R) .
$$

The dependence of the separation parameter $R$ on $a$ can be calculated as follows. Multiplying equation (6) by $\phi^{\prime}$ and integrating from $r=0$ to $r=\infty$, we get

$$
\frac{1}{2} \phi^{\prime}(0)^{2}-\sin ^{2}(\phi(0))=\int_{0}^{\infty} \frac{2 r}{r^{2}+a^{2}} \phi^{\prime 2} \mathrm{~d} r
$$


In terms of the mechanical analogy this equation represents the balance between the initial energy of the fictitious particle and the energy lost by friction. Substituting (13) into (14) and assuming that $R$ and $a$ are large, we get at the leading order $16 \mathrm{e}^{-2 \sqrt{2} R}=\gamma / a^{2}$, where $\gamma$ is a constant. Thus,

$$
R \approx \frac{1}{\sqrt{2}} \ln (a) \text { for } a \gg 1
$$

Similar large- $a$ approximations can be given for $n$-kinks with larger $n$.

Small $\boldsymbol{a}$ approximation: changing variables to $\rho=r / a$ and $g(\rho)=\phi(r)$, and taking the limit $a \rightarrow 0$, we get the linear equation

$$
\frac{\mathrm{d}^{2} g}{\mathrm{~d} \rho^{2}}+\frac{2 \rho}{\rho^{2}+1} \frac{\mathrm{d} g}{\mathrm{~d} \rho}=0
$$

whose two linearly independent solutions are $g_{1}=1$ and $g_{2}=\arctan \rho$. Thus, for $a \ll 1$ the $n$-kink is approximated by

$$
\phi_{n}(r) \approx n\left(\frac{\pi}{2}+\arctan (r / a)\right)
$$

\section{Linear perturbations}

In this section we analyse linear stability of kinks $\phi_{n}(r)$. Let

$$
\phi(t, r)=\phi_{n}(r)+\left(r^{2}+a^{2}\right)^{-1 / 2} u(t, r),
$$

where the perturbation $u$ is assumed to be small. Plugging this into equation (3), dropping nonlinear terms in $u$, and assuming harmonic time dependence $u(t, r)=\mathrm{e}^{-\mathrm{i} \omega t} v(r)$, we get the eigenvalue problem for the one-dimensional Schrödinger operator

$$
L_{n} v \equiv\left(-\frac{\mathrm{d}^{2}}{\mathrm{~d} r^{2}}+2+V_{n}(r)\right) v=\omega^{2} v
$$

with the potential

$$
V_{n}(r)=-4 \sin ^{2} \phi_{n}(r)+\frac{a^{2}}{\left(r^{2}+a^{2}\right)^{2}}
$$

Let us first consider the case $n=1$. As discussed above, in this case the one-kink $\phi_{1}(r)$ tends for $a \rightarrow \infty$ to the sine-Gordon kink $H(r)$ given by (10) for which the corresponding linear stability operator is

$$
L=-\frac{\mathrm{d}^{2}}{\mathrm{~d} r^{2}}+2-4 \sin ^{2} H(r)=-\frac{\mathrm{d}^{2}}{\mathrm{~d} r^{2}}+2-\frac{4}{\cosh ^{2}(\sqrt{2} r)}
$$

This operator has a continuous spectrum $\omega^{2} \geqslant 2$ (the bottom $\omega^{2}=2$ is a resonance) and a single eigenvalue $\omega^{2}=0$ which is due to translation symmetry of the sine-Gordon equation; the associated normalised eigenfunction (zero mode) is given by

$$
v_{0}(r)=2^{-3 / 4} H^{\prime}(r)=\frac{2^{-1 / 4}}{\cosh (\sqrt{2} r)} .
$$


The absence of eigenvalues in the gap $(0,2)$ is believed to be intimately tied with the complete integrability of the sine-Gordon equation [16].

The operator $L_{1}$ can be viewed as a compact perturbation of $L$ so it has the same continuous spectrum $\omega^{2} \geqslant 2$ but the discrete spectra are different. We claim that $L_{1}>L$. To show this, let us observe that $\phi_{1}(r)>H(r)$ for $r>0$ (and by the reflection symmetry $0<\phi_{1}(r)<H(r)$ for $r<0$ ). This fact is evident within our mechanical analogy because the fictitious particle corresponding to $\phi_{1}$ is subject to friction while the one corresponding to $H$ moves without friction. To see this, note that $\phi_{1}(0)=H(0)=\pi / 2$ and $\phi_{1}^{\prime}(0)>H^{\prime}(0)$, hence $\phi_{1}(r)>H(r)$ for small $r>0$. In fact, this inequality holds for all $r>0$ because the $H$-particle cannot overtake the $\phi_{1}$-particle (since at the overtake point the $\phi_{1}$-particle would have smaller kinetic energy than the $H$-particle and could not reach the hilltop). Since $-\sin ^{2} \phi$ is decreasing for $\phi \in[0, \pi / 2]$ and increasing for $\phi \in[\pi / 2, \pi]$, it follows that $-\sin ^{2} \phi_{1}(r)>-\sin ^{2} H(r)$ for all $r$ which by (19) and (21) implies that $L_{1}>L$. Since $L$ has exactly one eigenvalue at $\omega^{2}=0$, it follows (see, e.g. corollary 4.11 on page 119 in [17]) that $L_{1}$ has no negative eigenvalues and at most one positive eigenvalue in the gap $(0,2)$.

To obtain a more quantitative information about the gap eigenvalue of $L_{1}$ for large values of $a$ we seek a perturbative solution of the eigenvalue problem (19) in the form

$$
v=v_{0}+\varepsilon^{2} v_{1}+\mathcal{O}\left(\varepsilon^{4}\right), \quad \omega^{2}=c \varepsilon^{2}+\mathcal{O}\left(\varepsilon^{4}\right),
$$

where $\varepsilon=1 / a \ll 1$. Inserting (11) and (23) into (19), at the zero order we obtain $L v_{0}=0$, while at the order $\varepsilon^{2}$ we get

$$
L v_{1}=c v_{0}+2 r v_{0}^{\prime}+4 \sin (2 H) \psi v_{0} .
$$

The right-hand side must be orthogonal to $v_{0}$ which yields

$$
c=1-4 \int_{-\infty}^{\infty} \sin (2 H(r)) \psi(r) v_{0}(r)^{2} \mathrm{~d} r .
$$

To calculate the above integral we differentiate equation (12)

$$
L \psi^{\prime}=4 \sin (2 H) H^{\prime} \psi+2\left(r H^{\prime}\right)^{\prime} .
$$

Taking the inner product with $v_{0}$ and integrating by parts we get

$$
4 \int_{-\infty}^{\infty} \sin (2 H(r)) \psi(r) v_{0}(r)^{2} \mathrm{~d} r=-\int_{-\infty}^{\infty} v_{0}(r)^{2} \mathrm{~d} r=-1 .
$$

Substituting this into (24) we obtain $c=2$.

As $a$ decreases, the potential well gets shallower and eventually becomes a barrier, as follows from the small- $a$ approximation of kinks (17); see figure 3. Accordingly, as shown in figure 4 , the eigenvalue $\omega^{2}$ migrates through the gap $(0,2)$ and disappears into the continuous spectrum for $a$ smaller than some critical value $a^{*}$ (for $a=a^{*}$ there is a resonance at the bottom of the continuous spectrum $)^{7}$. Numerically, we find that $a^{*} \approx 0.536$.

For a given $n \geqslant 2$ and sufficiently large $a$, it follows from the large- $a$ approximation of $n$-kinks that the potential has the form of $n$ wells equally separated by the distance $\propto \ln (a)$. Consequently, if $a$ is large enough there are $n$ gap eigenvalues

$$
0<\omega_{1}^{2}<\cdots<\omega_{n}^{2}<2 .
$$

\footnotetext{
${ }^{7}$ A similar behaviour of the gap eigenvalue was found for some geometric wave equations on the hyperbolic space [18].
} 

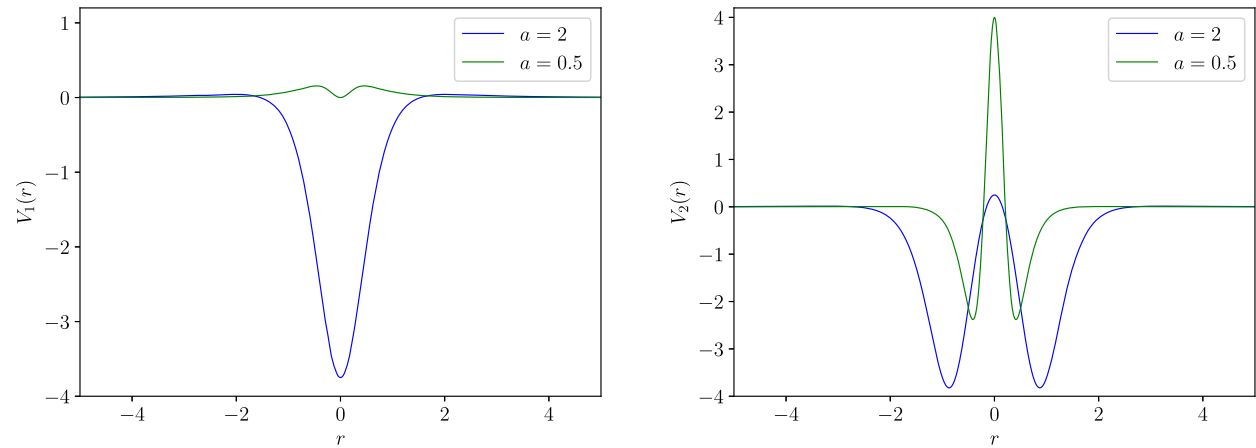

Figure 3. The potential $V_{n}(r)$ for $n=1,2$ and two values of $a$.

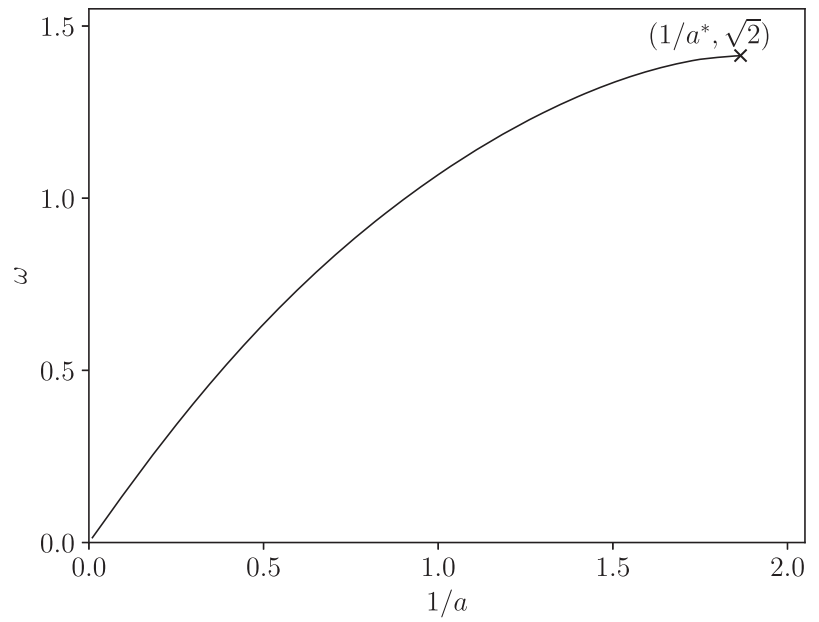

Figure 4. The frequency of the internal mode of the one-kink as a function of $1 / a$.

As $a$ decreases, the potential wells go up (see figure 3) and the gap eigenvalues disappear one by one into the continuous spectrum at certain critical values $a_{1}^{*}<a_{2}^{*}<\cdots<a_{n}^{*}$. For example, for $n=2$ we find numerically $a_{1}^{*} \approx 0.39$ and $a_{2}^{*} \approx 0.81$.

\section{Weakly nonlinear dynamics near the one-kink}

In this section we study solutions of equation (3) for initial data near the kink $\phi_{1}$. In terms of $u(t, r)$ defined in (18), equation (3) takes the form

$$
\ddot{u}+L_{1} u=f(u, r),
$$

where the linear operator $L_{1}$ is defined in (19) and the nonlinear term

$$
f(u, r)=\sqrt{r^{2}+a^{2}}\left[\sin \left(2 \phi_{1}\right)-\sin \left(2 \phi_{1}+\frac{2 u}{\sqrt{r^{2}+a^{2}}}\right)\right]+2 \cos \left(2 \phi_{1}\right) u
$$


is real-analytic in $u$ and $r$ (in what follows we suppress the dependence of $f$ on $r$ ). The Taylor series of $f(u)$ starts from the quadratic term:

$$
f(u)=\frac{2 \sin \left(2 \phi_{1}\right)}{\sqrt{r^{2}+a^{2}}} u^{2}+\frac{4 \cos \left(2 \phi_{1}\right)}{2\left(r^{2}+a^{2}\right)} u^{3}+\mathcal{O}\left(u^{4}\right) .
$$

As follows from (8), the coefficients in this expansion are decaying functions (exponentially for even powers of $u$ and algebraically for odd powers), which means that the nonlinear terms are spatially localised. This property, intimately related to the fact that our equation descends from higher dimensions, will play an important role in our analysis ${ }^{8}$.

We recall from the previous section that for $a<a^{*}$ the spectrum of the operator $L_{1}$ is purely continuous. In this case, the nonlinear term $f(u)$, due to its strong spatial localisation, does not affect the leading order asymptotic behaviour of small amplitude solutions. Consequently, such solutions decay as $t^{-3 / 2}$ for $t \rightarrow \infty$, which is the three-dimensional free linear dispersive decay or, equivalently, the one-dimensional linear dispersive decay in the presence of the rapidly decreasing potential (which has no bound states nor a resonance at the bottom of the continuous spectrum) [21, 22].

For the rest of this section we shall focus on the more interesting case $a>a^{*}$ where

$$
\operatorname{spec}\left(L_{1}\right)=\left\{\omega^{2}\right\} \cup[2,+\infty), \quad 0<\omega^{2}<2 .
$$

In what follows, the normalised eigenfunction associated to the eigenvalue $\omega^{2}$ is denoted by $v$, while the modes of the continuous spectrum are denoted by $\eta$. We decompose the solution as the orthogonal sum of the discrete and continuum modes of $L_{1}$

$$
u(t, r)=\alpha(t) v(r)+\eta(t, r), \quad \text { where } \quad\langle v, \eta\rangle=0 .
$$

Substituting this decomposition into (27) and projecting on the discrete and continuous components, using the projection operators $P f=\langle v, f\rangle v$ and $P^{\perp} f=f-\langle v, f\rangle v$ (where $\left.\langle v, f\rangle:=\int_{-\infty}^{+\infty} \bar{v}(r) f(r) \mathrm{d} r\right)$, we get a system

$$
\begin{aligned}
& \ddot{\alpha}+\omega^{2} \alpha=\langle v, f(\alpha v+\eta)\rangle, \\
& \ddot{\eta}+L_{1} \eta=P^{\perp} f(\alpha v+\eta) .
\end{aligned}
$$

This system describes interaction between the internal mode and radiation. When the nonlinearity is 'switched off', equations (31) and (32) decouple and the internal mode performs harmonic oscillations with frequency $\omega$. If the perturbation $u$ is small, the system is weakly coupled and the energy is slowly transferred from the internal mode to the continuum modes and then disperses to infinity. As the result, the amplitude of the internal mode decays asymptotically to zero and the solution converges (on any compact spatial interval) to the static solution $\phi_{1}$. The key mechanism of this relaxation process is the nonlinear resonance between the internal mode and the continuum. It was first described rigorously by Soffer and Weinstein in a seminal paper [15] (see also [23] for a formal construction, [24, 25] for ramifications, and [26] for a recent review). Below we will adapt their approach to our case, however in contrast to [15] we will not justify our formal calculations by error estimates (which would be quite technical). Instead, to feel confident that the results are true, in the next section we will verify them by numerical computations.

\footnotetext{
${ }^{8}$ We point out that the lack of spatial localisation of nonlinear interactions is one of the major difficulties in the studies of asymptotic stability of topological solitons in one spatial dimension, see [3, 19, 20].
} 
It is convenient to use the complex variable $z=\alpha+\frac{i}{\omega} \dot{\alpha}$ and rewrite equation (31) as the first-order ordinary differential equation

$$
\dot{z}+\mathrm{i} \omega z=\frac{\mathrm{i}}{\omega}\left\langle v, f\left(\frac{1}{2}(z+\bar{z}) v+\eta\right)\right\rangle
$$

We expand the nonlinearity in the formal power series in $z, \bar{z}$ and $\eta$

$$
f\left(\frac{1}{2}(z+\bar{z}) v+\eta\right)=\sum_{k+l+m \geqslant 2} f_{m k l} \eta^{m} z^{k} \bar{z}^{l}
$$

where indices run over nonnegative integers. The coefficients $f_{m k l}(r)$ are symmetric in the last two indices and can be read off from the Taylor series (29). Substituting this expansion into equations (33) and (32) we arrive at the system

$$
\begin{aligned}
& \dot{z}+\mathrm{i} \omega z=\frac{\mathrm{i}}{\omega} \sum_{k+l+m \geqslant 2}\left\langle f_{m k l} \eta^{m}, v\right\rangle z^{k} \bar{z}^{l}, \\
& \ddot{\eta}+L_{1} \eta=\sum_{k+l+m \geqslant 2} P^{\perp}\left(f_{m k l} \eta^{m}\right) z^{k} \bar{z}^{l} .
\end{aligned}
$$

In the following we introduce the symbol $\mathcal{O}_{p}(z, \eta)$ defined by

$$
\sum_{k+l+m \geqslant 2} c_{m k l} \eta^{m} z^{k} \bar{z}^{l}=\mathcal{O}_{p}(z, \eta) \quad \text { if } \quad \min \left\{k+l+2 m \mid c_{m k l} \neq 0\right\}=p,
$$

which incorporates a heuristic rule of thumb (to be justified a posteriori) that $\eta=\mathcal{O}\left(z^{2}\right)$. With this notation, those terms on the right-hand sides of equations (35) and (36) that do not involve $\eta$ are of the order $\mathcal{O}_{2}$, while those that involve $\eta$ are of the order $\mathcal{O}_{3}$.

Next, we make a near-identity coordinate transformation

$$
\eta=\tilde{\eta}+\sum_{k+l \geqslant 2} a_{k l} z^{k} \bar{z}^{l}
$$

where the coefficients $a_{k l}$ (which are symmetric) are functions of $r$. The purpose of this transformation is to eliminate the terms of order $\mathcal{O}_{2}$ on the right-hand side of equation (36). As we will see shortly, this change of variables is formal in the sense that, although a priori $\eta$ is decaying in space, the functions $a_{k l}$ in general do not decay. To justify rigorously the procedure described below one can consider (27) in weighted spaces consistent with (18), cf [22] or in the energy space following the arguments in [28-30]. A heuristic reason why the procedure works in the first place is that the system governing the dynamics of the internal modes is always localised by the projection on the eigenspace.

Substituting (38) into (36) we get

$$
\ddot{\tilde{\eta}}+L_{1} \tilde{\eta}+\sum_{k+l=2}\left(L_{1}-\omega^{2}(k-l)^{2}\right) a_{k l} z^{k} \bar{z}^{l}=\sum_{k+l=2} P^{\perp} f_{0 k l} z^{k} \bar{z}^{l}+\mathcal{O}_{3} .
$$

If we manage to remove the $\mathcal{O}_{2}$ terms then formally $\tilde{\eta}=\mathcal{O}_{3}$. To do so we impose the condition

$$
\left(L_{1}-(k-l)^{2} \omega^{2}\right) a_{k l}=P^{\perp} f_{0 k l}, \quad k+l=2 .
$$


For the combination of indices $(k, l)=(1,1)$, there is a unique real-valued solution $a_{11}(r)$ that decays for $|r| \rightarrow \infty$. We do not write it down because the only thing that matters is that this solution is real valued. For the combination $(k, l)=(2,0)$ we need to consider two cases: (i) $4 \omega^{2}>2$ and (ii) $4 \omega^{2}<2$ (we omit the nongeneric case $4 \omega^{2}=2$ which is more involved). We postpone the analysis of the case (ii) until afterwards and now we focus on the case (i). In this case, the frequency $2 \omega$ lies in the continuous spectrum of $L_{1}$ and therefore solutions of the homogeneous equation $\left(L_{1}-4 \omega^{2}\right) a=0$ are oscillatory at infinity. Among them there is a unique (complex) solution $a_{20}(r)$ that satisfies the outgoing boundary conditions for $|r| \rightarrow \infty$. Using the method of variation of parameters, this solution can be expressed in the form

$$
a_{20}(r)=\frac{\mathrm{i}}{2 \xi} k(r) \int_{-\infty}^{r} \bar{k}(s) P^{\perp} f_{020}(s) \mathrm{d} s+\frac{\mathrm{i}}{2 \xi} \bar{k}(r) \int_{r}^{\infty} k(s) P^{\perp} f_{020}(s) \mathrm{d} s,
$$

where $\xi=\sqrt{4 \omega^{2}-2}$ and $k(r)$ is the solution of the homogeneous equation satisfying the following outgoing boundary condition at $+\infty$

$$
k(r)=\mathrm{e}^{\mathrm{i} \xi r} m(r), \quad \lim _{r \rightarrow \infty} m(r)=1,
$$

and $\bar{k}(r)$ satisfies the corresponding outgoing boundary condition at $-\infty$. In (41) we used the Wronskian

$$
W[k(r), \bar{k}(r)]:=k(r) \bar{k}^{\prime}(r)-\bar{k}(r) k^{\prime}(r)=-2 \mathrm{i} \xi
$$

which follows from (42) and the fact that $\bar{k}(r)=k(-r)$ (what in turn follows from invariance of $L_{1}$ under reflections $\left.r \rightarrow-r\right)$.

Substituting (38) into (35) and using $\tilde{\eta}=\mathcal{O}_{3}$, we get

$$
\dot{z}+\mathrm{i} \omega z=\frac{\mathrm{i}}{\omega} \sum_{2 \leqslant k+l \leqslant 3}\left\langle v, f_{0 k l}\right\rangle z^{k} \bar{z}^{l}+\frac{\mathrm{i}}{\omega} \sum_{\substack{k+l=1 \\ p+q=2}}\left\langle v, f_{1 k l} a_{p q}\right\rangle z^{k+p} \bar{z}^{l+q}+\mathcal{O}_{4} .
$$

The key point is that all the dependence on $\tilde{\eta}$ on the right-hand side is contained in the term $\mathcal{O}_{4}$, hence up to the third order this equation is decoupled from the radiation equation. To factor out fast oscillations with frequency $\omega$ we let $z=\mathrm{e}^{-\mathrm{i} \omega t} Z$. Substituting this into (44) and dropping all nonresonant terms ${ }^{9}$ i.e. terms involving powers of $\mathrm{e}^{\mathrm{i} \omega t}$ (because such terms time-average to zero), we finally obtain the third order resonant approximation for $t>0^{10}$

$$
\dot{Z}=\frac{\mathrm{i}}{\omega}\left(\left\langle v, f_{021}\right\rangle+\left\langle v, f_{110} a_{11}\right\rangle+\left\langle v, f_{110} a_{20}\right\rangle\right) Z^{2} \bar{Z}
$$

The first two terms in the bracket are real, hence multiplying (45) by $\bar{Z}$ and taking the real part we get

$$
\frac{\mathrm{d}}{\mathrm{d} t}|Z|^{2}=-\Gamma|Z|^{4}, \quad \text { where } \quad \Gamma=\frac{2}{\omega}\left\langle v, f_{110} \operatorname{Im}\left(a_{20}\right)\right\rangle
$$

\footnotetext{
${ }^{9}$ A rigorous justification of this procedure, also called normal form transformation, can be found in [27].

${ }^{10}$ We point out that equation (45) is not invariant under the time reversal $t \rightarrow-t$. The arrow of time was selected by the outgoing boundary conditions imposed on $a_{20}$.
} 
The coefficient $\Gamma$ can be calculated as follows. From (29) and (30) we find

$$
f_{110}=\frac{2 \sin \left(2 \phi_{1}\right)}{\sqrt{r^{2}+a^{2}}} v \quad \text { and } \quad f_{020}=\frac{\sin \left(2 \phi_{1}\right)}{2 \sqrt{r^{2}+a^{2}}} v^{2}=\frac{1}{4} f_{110} v .
$$

Since $f_{020}(r)$ is an odd function while $v(r)$ is even, it follows that $P^{\perp} f_{020}=f_{020}$, and from (41) we get

$$
\operatorname{Im}\left(a_{20}\right)=\frac{1}{2 \xi} \operatorname{Re}\left(k(r) \int_{-\infty}^{\infty} \bar{k}(s) f_{020}(s) \mathrm{d} s\right)
$$

Inserting this expression into formula (46) and using (47), we finally obtain

$$
\Gamma=\frac{1}{\xi \omega}\left|\left\langle k, \frac{\sin \left(2 \phi_{1}\right) v^{2}}{\sqrt{r^{2}+a^{2}}}\right\rangle\right|^{2},
$$

hence $\Gamma \geqslant 0$. Generically $\Gamma$ is strictly positive ${ }^{11}$ and then equation (46) gives

$$
|Z| \sim \Gamma^{-\frac{1}{2}} t^{-\frac{1}{2}} \quad \text { as } \quad t \rightarrow \infty .
$$

The purely imaginary terms on the right-hand side of equation (45) determine the phase of $Z$. Returning to the amplitude $\alpha=\frac{1}{2}\left(\mathrm{e}^{-\mathrm{i} \omega t} Z+\mathrm{e}^{\mathrm{i} \omega t} \bar{Z}\right)$, we get the asymptotic behaviour

$$
\alpha(t) \sim \Gamma^{-\frac{1}{2}} t^{-\frac{1}{2}} \cos (\omega t+\theta(t)), \quad \text { where } \quad \theta(t)=\mathcal{O}(\ln t),
$$

hence as the amplitude of the internal mode decays asymptotically to zero, its frequency tends to the linear frequency $\omega$ (in other words, no frequency shift or 'memory' effect occurs).

Now, we return to the case (ii) $4 \omega^{2}<2$. In this case the frequency $2 \omega$ generated by the quadratic term is below the continuous spectrum so there is no resonant damping present at the third perturbative order (technically, in this case the solution $a_{20}$ of equation (40) is real and therefore the right-hand side of equation (45) is purely imaginary). One needs to go to higher orders to see the damping. Let $N$ be a positive integer such that

$$
N^{2} \omega^{2}<2<(N+1)^{2} \omega^{2}
$$

hence $(N+1) \omega$ is the lowest multiple of the frequency $\omega$ that lies in the continuous spectrum. The case (i) discussed above corresponds to $N=1$. For $N \geqslant 2$ we iterate the near-identity transformation (38) $N$ times to eliminate terms of order $N+1$ in the radiation equation. As the result of this iteration, the internal mode equation decouples from radiation up to order $\mathcal{O}_{2 N+1}$. By the same reasoning as above, this yields the resonant approximation of order $\mathcal{O}_{2 N+1}$ for the internal mode equation

$$
\dot{Z}=\sum_{1 \leqslant l<N} c_{l} Z^{l+1} \bar{Z}^{l}+c_{N} Z^{N+1} \bar{Z}^{N}
$$

where the coefficients $c_{l}$ with $l<N$ are purely imaginary, while $\operatorname{Re}\left(c_{N}\right) \leqslant 0$. Thus,

$$
\frac{\mathrm{d}}{\mathrm{d} t}|Z|^{2}=2 \operatorname{Re}\left(c_{N}\right)|Z|^{2 N+2},
$$

\footnotetext{
${ }^{11}$ This genericity condition is sometimes referred to as the Fermi golden rule [15, 31], which goes back to Dirac's theory of radiation in quantum mechanics [32].
} 
which gives (assuming that $\operatorname{Re}\left(c_{N}\right)$ is strictly negative) for $t \rightarrow \infty$

$$
|Z| \sim C_{N} t^{-\frac{1}{2 N}}, \quad \text { where } \quad C_{N}=\left(2 N\left|\operatorname{Re}\left(c_{N}\right)\right|\right)^{-\frac{1}{2 N}} .
$$

Assuming that the coefficient $c_{1}$ in (53) is nonzero, the amplitude of the internal mode behaves asymptotically as

$$
\alpha(t) \sim C_{N} t^{-\frac{1}{2 N}} \cos (\omega t+\theta(t)), \quad \text { where } \quad \theta(t)=\mathcal{O}\left(t^{\frac{N-1}{N}}\right) .
$$

Having the formulae for $\alpha(t)$, we now return to the radiation field $\eta(t, r)$. The asymptotic behaviour of $\eta(t, r)$ for $t \rightarrow \infty$ is determined by the quadratic term in equation (36), hence to the leading order we have

$$
\ddot{\eta}+L_{1} \eta \simeq P^{\perp}\left(\alpha^{2} f_{2} v^{2}+2 \alpha f_{2} v \eta+f_{2} \eta^{2}\right),
$$

where $f_{2}=\frac{2 \sin \left(2 \phi_{1}\right)}{\sqrt{r^{2}+a^{2}}}$ is the coefficient of the quadratic term in (29). All terms on the right-hand side are exponentially localised in space and the dominant contribution comes from the first term which behaves as $\alpha^{2}$. Inserting $\alpha(t) \sim t^{-1 / 2 N}$ and noting that the solution of the homogeneous equation decays as $t^{-3 / 2}$ [22], we conclude that $\eta(t, r) \sim t^{-1 / N}$. This heuristic argument justifies a posteriori that $\eta=\mathcal{O}\left(z^{2}\right)$, as claimed above.

\section{Numerical evidence}

In this section we solve equation (3) numerically using the hyperboloidal formulation of the initial value problem [33]. As in [8] we define new coordinates

$$
s=\frac{t}{a}-\sqrt{\frac{r^{2}}{a^{2}}+1}, \quad y=\arctan \left(\frac{r}{a}\right) .
$$

The hypersurfaces of constant $s$ are 'hyperboloidal', that is they are spacelike hypersurfaces that approach the 'left' and 'right' future null infinities of the wormhole spacetime along the outgoing null cones. In terms of the coordinates $(s, y)$ and $h(s, y)=\phi(t, r)$ equation (3) takes the form

$$
\partial_{s}^{2} h+2 \sin y \partial_{s} \partial_{y} h+\frac{1+\sin ^{2} y}{\cos y} \partial_{s} h=\cos ^{2} y \partial_{y}^{2} h-a^{2} \frac{\sin (2 h)}{\cos ^{2} y} .
$$

We solve this equation for smooth initial data of degree $n$

$$
h(0, y)=\alpha(y), \quad \partial_{s} h(0, y)=\beta(y),
$$

where the functions $\alpha(y)$ and $\beta(y)$ tend exponentially to $\alpha\left(-\frac{\pi}{2}\right)=0, \alpha\left(\frac{\pi}{2}\right)=n \pi$ and $\beta\left( \pm \frac{\pi}{2}\right)=0$. In this formulation, the $n$-kink denoted by $h_{n}(y)$ satisfies the boundary conditions $h_{n}\left(-\frac{\pi}{2}\right)=0, h_{n}\left(\frac{\pi}{2}\right)=n \pi$. No boundary conditions are imposed because the principal part of equation (59) degenerates to $\partial_{s}\left(\partial_{s} \pm 2 \partial_{y}\right) h$ at the endpoints $y= \pm \pi / 2$, hence there are no ingoing characteristics at the boundaries ${ }^{12}$.

\footnotetext{
${ }^{12}$ We remark that for massless fields considered in [8] there was an outflow of energy defined on hyperboloida slices through the boundaries due the outgoing radiation. In the case at hand, the boundaries do not participate in the evolution, and hence the energy is conserved, because the group velocity of waves is strictly less than one.
} 


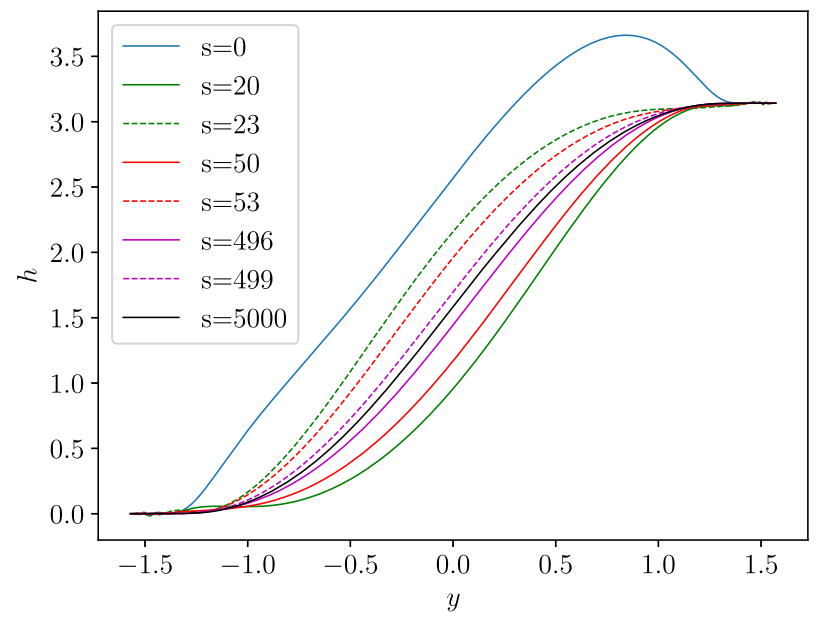

Figure 5. The snapshots of $h(s, y)$ for the initial data (63). The solution converges to the one-kink $h_{1}(y)$ (solid black line) in an oscillatory manner.

Following $[8,33]$ we define the auxiliary variables

$$
q=\partial_{y} h \quad \text { and } \quad p=\partial_{s} h+\sin y \partial_{y} h,
$$

and rewrite equation (59) as the first order symmetric hyperbolic system

$$
\begin{aligned}
& \partial_{s} h=p-q \sin y, \\
& \partial_{s} q=\partial_{y}(p-q \sin y), \\
& \partial_{s} p=\partial_{y}(q-p \sin y)+2 \tan y(q-p \sin y)-a^{2} \frac{\sin (2 h)}{\cos ^{2} y} .
\end{aligned}
$$

The initial data (60) translate to

$$
h(0, y)=\alpha(y), \quad q(0, y)=\alpha^{\prime}(y), \quad p(0, y)=\beta(y)+\sin y \alpha^{\prime}(y) .
$$

We solve this system numerically using the method of lines with a fourth-order Runge-Kutta time integration and eighth-order spatial finite differences. One-sided stencils are used at the boundaries. Kreiss-Oliger dissipation is added in the interior in order to reduce unphysical high-frequency noise. To suppress violation of the constraint $q-\partial_{y} h=0$, we add the term $\epsilon\left(q-\partial_{y} h\right)$ with a small negative $\epsilon$ to the right-hand side of equation (61b).

For any initial data of degree $n$, we find that the solution $h(s, y)$ converges pointwise to the $n$-kink $h_{n}(y)$ as $s \rightarrow \infty$. In the following, we focus on solutions of degree one and illustrate the results of numerical computations for sample initial data of the form (figure 5)

$$
\alpha(y)=h_{1}(y)+\mathrm{e}^{-\frac{1}{4} \tan ^{2} y}, \quad \beta(y)=0 .
$$

The convergence rates for several values of $a$ are depicted in figure 6 .

The results are in accord with the formulae (51) and (56) for the decay of the internal mode, and verify the decay rate $s^{-3 / 2}$ when the internal mode is absent. We emphasise that both the 


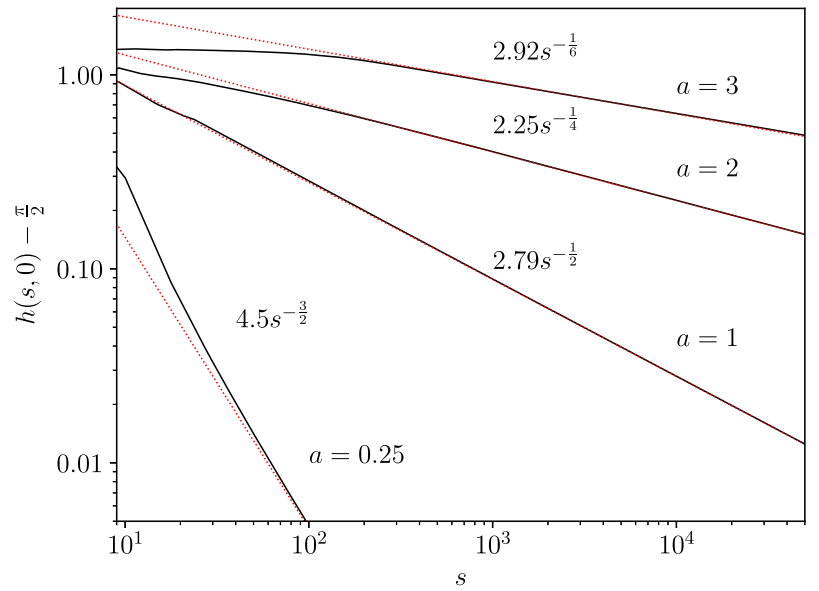

Figure 6. Amplitudes of perturbations evaluated at $y=0$ in the evolution of initial data (63) for different values of the wormhole radius: $a=0.25$ (no gap eigenvalue) and $a=1$ ( $\omega=1.0682), a=2(\omega=0.6345), a=3(\omega=0.4455)$, corresponding to $N=1,2,3$ in (52), respectively. The red dashed lines depict analytic predictions with empirically fitted coefficients.
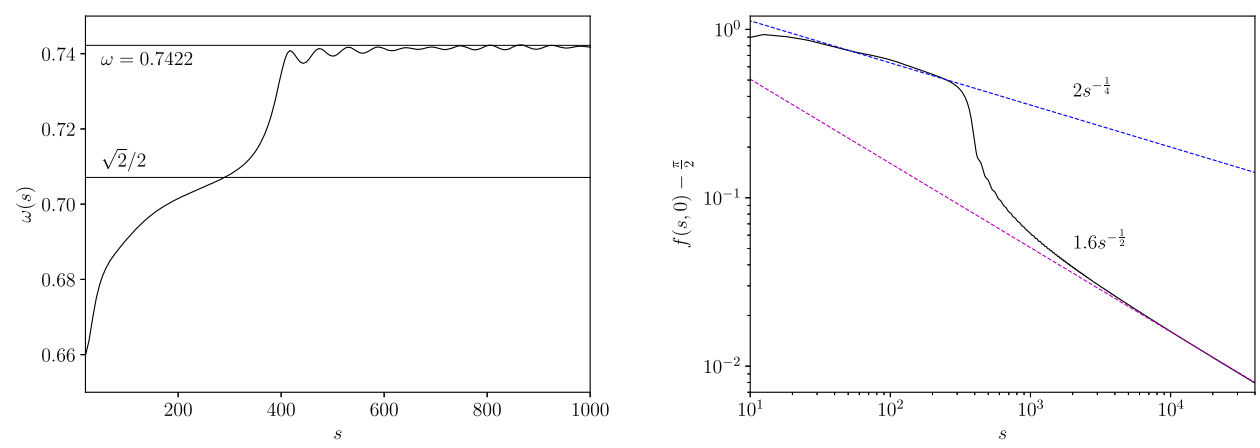

Figure 7. The effective frequency (left panel) and the amplitude (right panel) in the evolution of initial data (63) for $a=1.65$. The late-time small oscillations of the frequency are believed to be due to nonresonant interactions.

decay rates and the coefficients are universal (i.e. independent of initial data). In the $N=1$ case the coefficient 2.79 obtained from the empirical fit agrees (to three decimal places) with $\Gamma^{-1 / 2}$ calculated from formula (49). This excellent quantitative agreement between analytic and numerical results makes us feel confident that both computations are correct.

For the evolutions depicted in figure 6 the frequencies of internal modes are well separated from the threshold values $\sqrt{2} /(N+1)$. If the frequency of the internal mode is near a threshold value, an intermediate dynamics is more complicated. This is illustrated in figure 7 where we plot the effective frequency $\omega(s)$ (computed from the distances of subsequent maxima of oscillations) and amplitude of perturbation for $a=1.65$ for which $\omega=0.7422$ is a little above the $N=1$ threshold value $\sqrt{2} / 2 \approx 0.7071$. Initially, the effective frequency is below $\sqrt{2} / 2$ and the amplitude decays approximately as $s^{-1 / 4}$ (as in the $N=2$ case). For later times, the 
effective frequency increases above the threshold and concurrently the decay rate of the amplitude undergoes a transition to the asymptotic rate $s^{-1 / 2}(N=1$ case).

To summarise, the sine-Gordon equation on the wormhole is a rich model for developing understanding of the asymptotic stability of topological solitons with internal modes. In this work we focused mainly on dynamics of perturbations of the one-kink. It would be interesting to generalise the weakly nonlinear perturbation analysis from section 4 to $n$-kinks with multiple internal modes. We leave this to future work.

\section{Acknowledgments}

We acknowledge helpful conversations with Gary Gibbons during the early stage of this project. This work was supported in part by the National Science Centre Grant No. 2017/26/A/ST2/00530 (to PB), STFC consolidated Grant No. ST/P000681/1 (to MD), FONDECYT 1170164 and CMM Conicyt PIA AFB170001 (to MK).

\section{Appendix}

We describe here how we computed the coefficients $c_{n}$ in the asymptotic expansion (8). Since the solutions $\phi_{n}(r)$ of equation (6) are symmetric, it is sufficient to consider the asymptotic behaviour at one end, say $r \rightarrow \infty$

$$
n \pi-\phi_{n}(r) \sim \frac{c_{n}}{r} \mathrm{e}^{-\sqrt{2} r} .
$$

Let us observe that for large values of $r$ we have

$$
n \pi-\phi_{n}(r)=c_{n} \phi_{\mathrm{L}}(r)+\mathcal{O}\left(\mathrm{e}^{-3 \sqrt{2} r}\right),
$$

where $\phi_{\mathrm{L}}(r)$ is the solution of the linearised equation

$$
\phi_{\mathrm{L}}^{\prime \prime}+\frac{2 r}{r^{2}+a^{2}} \phi_{\mathrm{L}}^{\prime}-2 \phi_{\mathrm{L}}=0,
$$

such that

$$
\phi_{\mathrm{L}}(r) \sim \frac{1}{r} \mathrm{e}^{-\sqrt{2} r} \text { for } r \rightarrow \infty .
$$

Equation (66) has two regular singular points $r= \pm a$ i and the irregular singularity at $r=\infty$ and the general solution can be expressed in terms of confluent Heun functions [34]

$$
\phi_{\mathrm{L}}=C_{1} \operatorname{HeunC}\left(0,-\alpha, 0, \gamma, \delta,-r^{2} / a^{2}\right)+C_{2} \operatorname{HeunC}\left(0, \alpha, 0, \gamma, \delta,-r^{2} / a^{2}\right),
$$

where $\alpha=\frac{1}{2}, \gamma=\frac{1}{2} a^{2}, \delta=-\frac{1}{2} a^{2}+\frac{1}{4}$. In order to find the coefficients $C_{1}$ and $C_{2}$ such that $\phi_{\mathrm{L}}$ satisfies (67) we proceed in two steps. In the first step, we temporarily set $C_{1}=1$ and determine $C_{2}$ numerically by solving the equation $\phi_{\mathrm{L}}(r)=0$ for some large value of $r$, say $r=60$. This computation has to be performed with very high precision (up to 60 decimal places) which is possible in Maple where the Heun functions are tabulated. Having that, in the second step, we compute numerically the limit $\beta=\lim _{r \rightarrow \infty} r \mathrm{e}^{\sqrt{2} r} \phi_{\mathrm{L}}(r)$. This is done by evaluating $r_{j} \mathrm{e}^{\sqrt{2} r_{j}} \phi_{\mathrm{L}}\left(r_{j}\right)$ for an increasing series of $r_{j}$ (say from $r_{1}=20$ to $r_{10}=30$ ) and using the 
Shanks transformation to accelerate the convergence. Rescaling the coefficients $C_{k} \mapsto C_{k} / \beta$ we get the solution $\phi_{\mathrm{L}}(r)$ satisfying (67). Finally, taking the numerical solution $\phi_{n}(r)$ obtained by the shooting method we compute the coefficients $c_{n}$ using the formula

$$
c_{n} \approx \frac{n \pi-\phi_{n}\left(r_{0}\right)}{\phi_{\mathrm{L}}\left(r_{0}\right)}
$$

where $r_{0}$ is small enough, say $r_{0}=6$, so that the value $\phi_{n}\left(r_{0}\right)$ is accurate.

\section{References}

[1] Soffer A 2007 Soliton dynamics and Scattering ICM-2006 Conference Book

[2] Tao T 2009 Why are solitons stable? Bull. Am. Math. Soc. 46 1-33

[3] Kowalczyk M, Martel Y and Muñoz C 2017 On asymptotic stability of nonlinear waves Séminaire Laurent Schwartz-EDP et applications (2016-2017) vol 18 p 27

[4] Eckhaus W and Schuur P 1983 The emergence of solitons of the Korteweg-de vries equation from arbitrary initial conditions Math. Methods Appl. Sci. 5 97-116

[5] Schuur P C 1986 Asymptotic Analysis of Soliton Problems (An Inverse Scattering Approach) Lecture Notes in Mathematics vol 1232 (Berlin: Springer)

[6] Duyckaerts T, Kenig C and Merle F 2013 Classification of the radial solutions of the focusing, energy-critical wave equation Camb. J. Math. 175-144

[7] Kenig C, Lawrie A, Liu B and Schlag W 2015 Stable soliton resolution for exterior wave maps in all equivariance classes Adv. Math. 285 235-300

[8] Bizoń P and Kahl M 2015 Wave maps on a wormhole Phys. Rev. D 91065003

[9] Ellis H G 1973 Ether flow through a drainhole: a particle model in general relativity J. Math. Phys. 14104

[10] Bronnikov K 1973 Scalar-tensor theory and scalar charge Acta Phys. Pol. B 4 251-66

[11] Morris M S and Thorne K S 1988 Wormholes in spacetime and their use for interstellar travel: a tool for teaching general relativity Am. J. Phys. $\mathbf{5 6} 395$

[12] Rodriguez C 2018 Soliton resolution for equivariant wave maps on a wormhole Commun. Math. Phys. 359 375-426

[13] Rodriguez C 2019 Soliton resolution for corotational wave maps on a wormhole Int. Math. Res. Not. 2019 4603-706

[14] Waterhouse A 2019 The $\phi^{4}$ kink on a wormhole spacetime (arXiv:1908.09650)

[15] Soffer A and Weinstein M I 1999 Resonances, radiation damping and instabilitym in Hamiltonian nonlinear wave equations Invent Math. 136 9-74

[16] Kivshar Y S, Pelinovsky D E, Cretegny T and Peyrard M 1998 Internal modes of solitary waves Phys. Rev. Lett. 80 5032-5

[17] Teschl G 2009 Mathematical Methods in Quantum Mechanics (With Applications to Schrödinger Operators) (Providence, RI: American Mathematical Society)

[18] Lawrie A, Oh S-J and Shahshahani S 2016 Gap eigenvalues and asymptotic dynamics of geometric wave equations on hyperbolic space J. Funct. Anal. 271 3111-61

[19] Lindblad H, Luhrmann J and Soffer A 2020 Asymptotics for 1D Klein-Gordon equations with variable coefficient quadratic nonlinearities (arXiv:2006.00938)

[20] Germain P and Pusateri F 2020 Quadratic Klein-Gordon equations with a potential in one dimension (arXiv:2006.15688)

[21] Schlag W 2006 Spectral theory and nonlinear partial differential equations: a survey Discrete Continuous Dyn. Syst. 15 703-23

[22] Egorova I E, Kopylova E A, Marchenko V A and Teschl G 2016 Dispersion estimates for onedimensional Schrödinger and Klein-Gordon equations revisited Uspekhi Mat. Nauk 71 3-26

[23] Pelinovsky D E, Kivshar Y S and Afanasjev V V 1998 Internal modes of envelope solitons Physica D $116121-42$

[24] Bambusi D and Cuccagna S 2011 On dispersion of small energy solutions of the nonlinear Klein Gordon equation with a potential Am. J. Math. 133 1421-68

[25] An X and Soffer A 2020 Fermi's golden rule and $\mathrm{H}^{1}$ scattering for nonlinear Klein-Gordon equations with metastable states Discrete Continuous Dyn. Syst. A 40 331-73 
[26] Cuccagna S and Maeda M 2020 A survey on asymptotic stability of ground states of nonlinear Schrödinger equations II (arXiv:2009.00573)

[27] Bambusi D 2011 Asymptotic stability of ground states in some Hamiltonian PDEs with symmetry Commun. Math. Phys. 305 279-331

[28] Kowalczyk M, Martel Y and Muñoz C 2017 Kink dynamics in the $\phi^{4}$ model: asymptotic stability for odd perturbations in the energy space J. Am. Math. Soc. 30 769-98

[29] Kowalczyk M, Martel Y and Muñoz C 2019 Soliton dynamics for the 1D NLKG equation with symmetry and in the absence of internal modes (arXiv:1903.12460)

[30] Kowalczyk M, Martel Y, Muñoz C and van Den Bosch H 2020 A sufficient condition for asymptotic stability of kinks in general $(1+1)$-scalar field models (arXiv:2008.01276)

[31] Sigal I M 1993 Non-linear wave and Schrödinger equations Commun. Math. Phys. 153 297-320

[32] Dirac P A M 1927 The quantum theory of the emission and absorption of radiation Proc. R. Soc. Lond. A $114243-65$

[33] Zenginoğlu A 2008 A hyperboloidal study of tail decay rates for scalar and Yang-Mills fields Class. Quantum Grav. 25175013

[34] NIST digital library of mathematical functions http://dlmf.nist.gov/ 